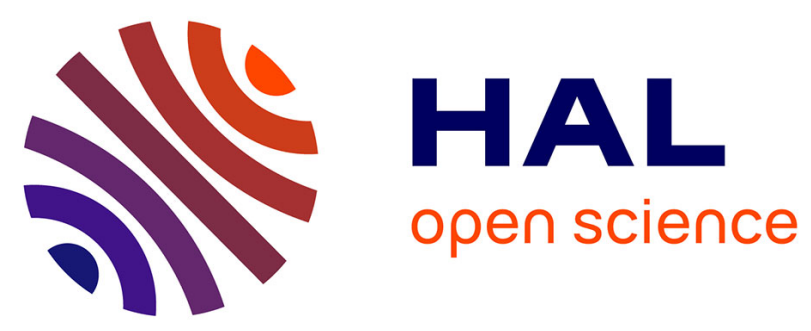

\title{
Towards an Integrated Approach of Real-Time Coordination for Multi-Agent Systems
}

\author{
Ghulam Mahdi, Abdelkader Gouaich, Fabien Michel
}

\section{To cite this version:}

Ghulam Mahdi, Abdelkader Gouaich, Fabien Michel. Towards an Integrated Approach of Real-Time Coordination for Multi-Agent Systems. 4th International Symposium on Agent and Multi-Agent Systems: Technologies and Applications (KES-AMSTA), Jun 2010, Gdynia, Poland. pp.253-262, 10.1007/978-3-642-13480-7_27. lirmm-00520972

\section{HAL Id: lirmm-00520972 https://hal-lirmm.ccsd.cnrs.fr/lirmm-00520972}

Submitted on 11 Dec 2019

HAL is a multi-disciplinary open access archive for the deposit and dissemination of scientific research documents, whether they are published or not. The documents may come from teaching and research institutions in France or abroad, or from public or private research centers.
L'archive ouverte pluridisciplinaire HAL, est destinée au dépôt et à la diffusion de documents scientifiques de niveau recherche, publiés ou non, émanant des établissements d'enseignement et de recherche français ou étrangers, des laboratoires publics ou privés. 


\title{
Towards an Integrated Approach of Real-Time Coordination for Multi-agent Systems
}

\author{
Ghulam Mahdi, Abdelkader Gouaïch, and Fabien Michel \\ LIRMM, Université Montpellier II, \\ UMR 5506 - CC477, 161 rue Ada, \\ 34095 Montpellier Cedex 5, France \\ \{ghulam.mahdi, abdelkader.gouaich,fmichel\}@lirmm.fr \\ http://www.lirmm.fr
}

\begin{abstract}
Real-time computations in multi-agent systems have been studied from different perspectives of reasoning, message passing, resource management and negotiations. Separate treatment of all these perspectives may result in performance amelioration in one dimension while over-passing others. Here we propose an integrated "real-time" mechanism where all dimensions of temporal behavior are suggested in one unified model.

Here in this position paper, we argue for an integrated and comprehensive treatment of "real-time" issue under the title of coordination. Our position regarding real-time agent coordination would result in overall performance betterment and overhead lessening, if all sub-processes are made to respect "notion of time" in design. We analyze current approaches and options as well as present an outline for an integrated and comprehensive view of "real-time coordination".
\end{abstract}

\section{Introduction}

Coordination has remained a key concern in multi-agent systems studies in order to achieve globally coherent results 181920. Some works even argue on the separation of treatment for computation and coordination as two distinct and orthogonal dimensions of all useful computing inferring explicit treatment of coordination 21.

Recently real-time coordination has been introduced in the fields of robotics [17], traffic management [16] and multimedia systems [22]. As multi-agent systems are being proposed for time-sensitive domains, we propose a similar study to be carried out from the perspective of real-time systems. Here in this position paper, we argue that there is a need of an integrated and comprehensive view of "realtime" phenomenon when suggested for multi-agent systems. The idea is to utilize lessons learned from earlier studies in multi-agent systems carried out from different perspectives of "real-time" processing and suggest an integrated view covering all these aspects. The objective of this article is to shed some light on an integrated and comprehensive view of real-time coordination presenting all aspects of multiagent systems processing as sub-processes being followed by time constraints. The 
rest of this article is structured as follows: the next section introduces some motivations from earlier works and real world scenarios for investigating coordination issues; then the following section is about characterizing some key dimensions of coordination and influence of "real-time" on them; later we discuss which factors will contribute to our integrated view of coordination then we present some guidelines as our criteria for measuring integrated real-time coordination; finally a discussion presents overall features of such integrated view and conclusions of our study.

\section{Motivations}

Real-time systems and multi-agents systems have individually contributed to many complex, heterogeneous and diversed real-world applications even before joining hands to be applied in domains particularly known for distributed, timecritical and autonomous features. A transfusion of both disciplines has shown quite interesting results in diverse domains ranging from sensor networks [1] to virtual classrooms [2] and from e-commerce applications 3 ] to soccer robots [4]. Apart from these applicative studies of multi-agent and real-time systems, many works have tried to define, develop and implement efficient agent models presenting features of both disciplines (like [56]); some others have focused on the frameworks simulating such real-time agents [7]; even others align to developing joint architectures for such type of agents [89].

However a fundamental issue of coordinating multi-agent systems at real-time constraints has remained largely unaddressed, even if some of works which have tried to address the agent coordination are limited to focus on only one aspect sub-process instead of addressing the issue in an integrated manner. Our motivations to address the problem has theoretical as well applicative inspirations. From the perspective of applications, most of real-time multi-agent systems are functioning in resource-constrained environments. In such domains of applications, usually agents share a single precious resource for a limited time to an agent so there is a need of coordinating the system processes for effective utilization of the resources in given time. Examples of such limited resources include single road, bridge, clean air, energy resource in the domains of virtual emergencies, hospital rooms and virtual rescue systems. In other domains, where it seems that there isn't apparent inadequacy of resources as satellites, printers, servers and network traffic but exorbitant nature of these resources leads for sharing distribution of the tasks and the common resources for a limited time. When there is involvement of multiple entities for sharing a resource or distribution of tasks there is an inherent element of competitiveness. In case of resource management "real-time coordination" may have more critical job to do as all comprising autonomous agents try to get the maximum of the resource share for most of times and in task distribution for common goals- each agent may have a "get the least share of the common task" tendency and above all ensuring due share of resources to all the agents and timely completion of the tasks. Theoretical motivations for our study come from the realization that coordination problem at real-time level has not been viewed in agent studies. This "position" nature of work examines 
agent coordination studies at different levels and suggests an integrated as well comprehensive framework for efficient "real-time coordination".

The time factor is so immersely embedded in the studies of multi-agent systems that most of coordination studies have an intrinsic treatment if not the explicit one as we are mentioning below. On the applications side, we realize that a lack of an efficient coordination mechanism in health services, virtual emergencies, distributed weather forecasting and traffic control may lead to performance degradation if not catastrophic results.

\section{Three Dimensions of Agent Coordination}

Edmund Durfee(in [1011]) characterizes three dimensions of scaling up agent coordination namely agent population, task environment and the solution properties. He argues that an efficient coordination mechanism needs to address all three dimensions of coordination. Here we briefly discuss all three dimensions and present our perspective that how temporal behavior influences all of three dimensions of coordination.

\subsection{Agent Population Properties}

Agent population properties include number of participating agents; complexity i.e. how complex they are in their architectures and internal reasoning mechanisms; and heterogeneity of communication languages, ontological and internal architectures. Here coordination without having temporal constraints may lead to serious delays and performance degradation as the increase in number of agents, diversity and complexity would also require an increase in coordination time, if it is let to function on its pace.

\subsection{Task Environment Properties}

Task environment properties are about defining how an environment influences overall coordination task. Main characteristics of agent environment include the degree of interaction between participating agents, environment dynamics and distributivity of agents in the environment obviously require a coordination solution having "real-time" factor . In other case resulting coordination may turn not only ineffective due to normal pace of interaction but also irrelevant due to environmental dynamics and distributivity of agents.

\subsection{Solution Properties}

Solution of any proposed coordination mechanism have to consider how the resulting coordination is efficient in terms of quality, how much overhead is there in terms of communication, computation and spent time along with robustness of the solution. Here real-time requirements are directly involved in terms of time spent and the resulting coordination in in due time along with minimum overhead of time resources. 
Having a brief idea about the implications of "time factor" in agent coordination, now we individually describe the factors involved in amelioration of "time factor" in agent computations. By description of these "performance contributers" we argue that these processes may be viewed as constituting sub-processes in global agent coordination process for an integrated view of "real-time coordination" in multi-agent systems. Here we describe different studies on these "performance contributers" and their role in integrated and comprehensive understanding of "realtime coordination" of multi-agent systems.

\section{Notion of Time in Agent Coordination}

\subsection{Time in Message Passing}

Assuming agents process incoming messages atomically as soon as they receive them( or buffered in the message inbox), we need to take care of how much time it takes to deliver a message. Having timing constraints on the delivery of messages may play a substantial role in managing temporal behavior of the overall system. Embracing monitors that ensure timely dispatch of the messages do not have to come in conflict with the timing constraints in message processing in a way that message delivery is not to breach the agent encapsulation of how and when the message is processed, rather it's sole concern would be about timely delivery of the incoming messages. Such message dispatch monitors may be provided in one of two forms: either, this monitoring mechanism would be made a part of agent message whose timing is maintained by the agent generating the message or some independent controllers which may have a check on message invocations and depending on later status of message invocations and agent constraints decides postponement or reordering of the messages in the message queue. Jamali et al. 13] suggest similar approach for multi-agent systems in resource allocation. Although the approach works quite finely, but its performance improvement is limited to resource management, in other words, it covers only one dimension of real-time performance in multi-agent systems.

\subsection{Time in Agent Reasoning}

Once a message is passed to the concerned agent, it may take time some time to read the message, evaluate the contract and subsequently reply in denial or follow the message contents. If time factor is not involved in such message processing or agent reasoning it would unnecessarily affect the agent performance resulting in delay of overall coordination process. Many works of Julien et al. (like [568]) are addressed on development, design and implementation of real-time agents without considering coordination as the main subject of studies. Here we need to make such models enough flexible with other real-time computations like the above mentioned ones. In absence of timing constraints the system processes the messages may take too much time and leading to affect the overall progress of the system. 


\subsection{Time in Resource Management}

Agents being part of open systems compete for resources due to sharing of independent computations. Such competition to acquire resources leads to functional and non-functional dependencies. Functional dependencies are about whether sufficient resources are available or not, how to acquire and release certain resources and how to deal with multiple requests of the same resource at the same time. By non-functional dependencies, we mean that availability (or at least information of unavailability ) of the required resources in certain time bounds. Such availability or unavailability information would be seen as an important factor in overall agent coordination. Here we need to manage autonomy of agents in a way that agents are not to be let to accumulate all the resources so here some type of resource management behavior is also recommended. Jamali et al. [13]'s work on real-time resource allocations is seminal on the subject that it not only ensures real-time in resource allocations but also handles excessive resource acquisitions problem common to agents based on actor model.

\subsection{Time in Negotiations}

Although agent coordination doesn't imply cooperation but many times coordination is seen as a co-operative process to maintain heterogeneous body of agents in an environment. Agent negotiations are used as a means to reach an accord through communications. Agent negotiations are usually seen as a compromising tool to mutual benefits of efficient resource usage and task distribution. Despite benefits of reaching an agreement, agent negotiations process is presumed as a costly and time consuming practice. When agent negotiations are left to work on their momentum it would not only delay the coordination process but also consume unnecessary resources. A model for real-time agent negotiations for sensor networks is presented in [14, another important works on the subject is suggested by Fatima et al. [15] but both of these works address negotiations to be constrained by time rather than directly treating it as real-time issue.

After a brief introduction of different real-time mechanisms in multi-agent systems, we return to our earlier proposition that coordination should be viewed as a meta-collection of different sub-processes based on universal guidelines covering all aspects of coordination. Here we present our criteria as a set of basic guidelines that we propose to be part of any coordination mechanism suggested to fully address real-time issue at all levels.

\section{Key Principles and Guidelines in Real-Time Agent Coordination}

Whether it is designing a coordination mechanism or developing and implementing a coordination model we obviously need certain guidelines and principles on which that particular solution would be based. The idea is to see coordination as a composition of different sub-processes and apply real-time constraints on all the 
constituent steps based on some criteria. Our vision of real-time is partially influenced by Douglas Jensen 12] for applying time constraints on all sub-activities and Soh [1]'s ideas on for setting up a criterion-based real-time systems. Here we briefly describe our set of guidelines for real-time coordination:

1. Agent coordination may be seen as a compound process involving all four sub-process viz a viz agents, resource management, negotiations and message passing. Here an efficient approach to realize real-time behavior would be the one which involves real-time in all sub-processes.

2. Agent coordination must be confined by time bounds. Take warbot (Unmanned systems in military applications playing a role in determining the success or failure of combat missions) agents as an example whose job is to coordinate with other agents before a missile enters into their covered territory . Even if an efficient coordination is achieved after the target reaches their covered zone, the coordinate would loose its applicability as well as appropriateness. As a result, the system is no longer useful for tracking the target. Similar applications widely exist in health systems, sensor networks and complex simulations where loosing time constituent profoundly affects the progress and purpose of a system.

3. All sub-processes of coordination should have a notion of promptness. As coordination activity may involve certain sub-tasks or steps like:message passing, responding to the messages, negotiating, etc. All these sub-steps must be performed with consideration to the time issues.

4. Coordination process should have minimum number of sub-activities. Coordination may involve multiple activities of negotiating to reach an accord, generating message, handling individual message s and responding received messages. Although the number of sub-processes may vary as per the situation, sensitivity and nature of coordination but one universal principle needs to respected is that as increase of the number of sub-processes is directly proportional to increase in time. The systems critical to time factors need to minimize number of such activities.

5. A coordination related message should have minimized contents. Message processing would be as time taking job as the contents of an individual message. Once a short message is received the agent would facilitate it in timely processing also if it needs to generate other messages having shorter contents would not halt its other processes.

6. Autonomous behavior in time message processing should be used. Many times dispatching a message takes time more than even message processing due to network congestion, bandwidth limitation and other issues. In such cases the agents should show a rational behavior by aborting that communication and move on for next message or agents instead of too much wait.

7. A coordination mechanism should be rapidly devised. As a scenario shows a need of coordination, the participating agent(s) should device how much time is alloted to the coordination, number of messages and other related prerequisites as soon as possible. Once these are decided agents can move for next steps. If chalking out the strategy takes much time, it will of course affect overall coordination process. 
8. A coordination model should have suitable mechanism to differentiate between different tasks. Coordination in multi-agent systems have a dynamic nature in a way that the participating agents may be involved in some other activities while busy in coordination for some task. Here there are two issues: if agents choose to notice every tiny activity of course it will delay the coordination process while going for the coordination process by ignoring all the activities may bring finally an irrelevant solution. Here we need to device a mechanism that in which cases agents have to respond to the other actions besides their coordination activities and when they have to ignore tiny activities.

9. Agent negotiations should occur within time constraints. Agent based coordination is often involved by negotiations to reach an accord. There has been various attempts to improve the negotiation languages, terminology and communication modes to achieve better communication but what we emphasize is to consider notion of "time" in the negotiation model.

10. Resource allocations should have a notion of time. Agent coordination frequently involves generation, consumption and freeing up resources for the computations. We need to emphasize that this important aspect of agent coordination should have some temporal behavior as it would affect overall coordination process.

11. Agent interactions should be time-bounded activity. Agent interactions are the means to interchange information with other agents, roles and environments. Emphasis on time bounds reminds the specification of temporal restrictions in these interactions. Here we need to specify interaction protocols with timing deadlines and expected influence on the quality of overall timing in the system.

\section{Discussion}

Multi-agent systems have been studied from different aspects of real-time processing, namely reasoning, message passing, resource management and negotiations. All these aspects of "real-time multi-agent systems" can be seen as "sub-processes" of a global and overall agent computation and coordination process. Such real-time distributed computation and coordination processes can be viewed as a composition of sub-processes (instead of a single agent process) where each sub-process coordinates with its comprising components along with other sub-systems at its stage while being part of the global coordination process. There are two approaches to see the real-time coordination in multi-agent systems:

1. Incorporating time constraints on individual processes and coordination of any of the individual process would of course bring amelioration in the performance of that system but not at the optimum level.

2. Setting up a meta approach of real-time as well as coordination in a way that the coordination is involved at all sub-processes' level which improves overall real-time performance of the system at global level. 
Our vision to see real-time agent coordination can be distincted from other coordination mechanisms following second approach of involving real-time at each level of computation and coordination. The approach may prove useful in understanding both coordination as well as real-time performance of multi-agent systems. Due to the differences in the architectures and performance measures of different systems we suggest an integrated treatment of "real-time" problem at the level of each sub-process as every sub-system incorporates notion of realtime. Human societies also adopt coordination mechanisms which may involve myriad sub-processes, at some extent seem even irrelevant but after all serving a global purpose. Like an office working procedure may adopt different procedures and sub-processes for their coordination and time constraints but after all it serves timely performance of the main objective.

Real-time multi-agent systems have special architectural foundations and design aspects current approaches in real-time agent systems were not set forth with those considerations therefore there is a performance as well as efficiency gap in effective agent coordination. Earlier studies on the subject have either dealt scalable agent coordination 1011] or particularizing coordination for different application domains (like [32]). As per our knowledge we haven't seen studies on real-time agent coordination which involves real-time in all sub-processes, although similar studies are carried out in robotics and communication domain from the perspective of coordination [16] and communication 17. Here our contribution comes in three folds: first, it suggests real-time treatment of agent systems at the level of coordination; and second, it sees coordination as an integrated process comprised of different sub-processes; and the third, it suggests real-time at every sub-process level of agent computations. Such integrated approach would result in overall performance betterment as well as overhead lessing in agent computations.

\section{Conclusion}

Here in this paper, we attempt to understand the peculiarities of real-time coordination for multi-agent systems. We feel that current approaches on the subject present serious concerns for understanding and applying coordination models in multi-agent systems. In this position paper, we have argued that real-time coordination has some different implications when viewed from a global perspective of "real-time" at different levels of multi-agent computation and coordination. We are of the view that, in order to support efficient coordination mechanisms for real-time multi-agent systems we need to understand the key differentiating factor that make real-time coordination different in multi-agent systems. We have discussed some key characteristics worthy to considered for devising any coordination mechanism for real-time multi-agent systems.

Our findings of the particular guidelines and constituting sub-processes would have an impact in developing the relevant and potential works in the field. Our position regarding the subject defines the distinguishing guidelines of real-time multi-agent systems which may be considered as starting points for devising efficient coordination schemes in such systems. The approach discussed here would 
let both multi-agent and real-time communities to see each other's requirements and prospectus in their domains. More precisely, the agent community to see coordination in multi-agent systems deal differently than it has been and the realtime community to take a more realistic picture about the agents' functionality and effectiveness in multi-agent systems. Clearly there is much left to be done. As a future perspective, we plan to work on our suggested "real-time"criteria based coordination along with designing and implementing this integrated model of agent coordination.

\section{References}

1. Soh, L., Tsatsoulis, C.: A Real-Time Negotiation Model and A Multi-Agent Sensor Network Implementation. Autonomous Agents and Multi-Agent Systems 11(3), 215-271 (2005)

2. Liu, X., Zhang, X., Soh, L.K., Al-Jaroodi, J., Jiang, H.: A distributed, multiagent infrastructure for real-time, virtual classrooms. In: Proceedings of the International Conference on Computers in Education (ICCE 2003), Hong Kong, pp. 2-5 (2003)

3. DiPippo, L.C., Fay-Wolfe, V., Nair, L., Hodys, E., Uvarov, O.: A real-time multiagent system architecture for e-commerce applications. In: Proceedings of the Fifth International Symposium on Autonomous Decentralized Systems, pp. 357-364. IEEE Press, Washington (2001)

4. Kim, J.H., Shim, H.S., Kim, H.S., Jung, M.J., Choi, I.H., Kim, J.O.: A cooperative multi-agent system and its real time application to robot soccer. In: Proceedings of the IEEE International Conference on Robotics and Automation, pp. 638-643. IEEE, USA (1997)

5. Julian, V., Botti, V.: Developing real-time multi-agent systems. Integrated Computer-Aided Engineering 11(2), 135-149 (2004)

6. Julian, V., Soler, J., Moncho, M.C., Botti, V.: Real-Time Multi-Agent System Development and Implementation. In: Frontiers in artificial intelligence and applications, Ourense, Espana, vol. 113, pp. 333-340 (2004)

7. Micacchi, C., Cohen, R.: A framework for simulating real-time multi-agent systems. Knowl. Inf. Syst. 17(2), 135-166 (2008)

8. Soler, J., Julian, V., Rebollo, M., Carrascosa, C., Botti, V.: Towards a real-time multi-agent system architecture. In: Proceedings of the First International Workshop on Challenges in Open Agent Systems, AAMAS 2002, Bologna, Italy (2002)

9. Carrascosa, C., Bajo, J., Julian, V., Corchado, J.M., Botti, V.: Hybrid multi-agent architecture as a real-time problem-solving model. Expert Syst. Appl. 34(1), 2-17 (2008)

10. Durfee, E.H.: Scaling Up Agent Coordination Strategies. Computer 34(7), 39-46 (2001)

11. Durfee, E.H.: Challenges to Scaling Up Agent Coordination Strategies. In: Wagner, T.A. (ed.) An Application Science for Multi-Agent Systems, pp. 113-132. Kluwer Academic Publishers, Dordrecht (2004)

12. Real-Time for the Real World, http://www.real-time.org

13. Jamali, N., Ren, S.: A layered architecture for real-time distributed multi-agent systems. ACM SIGSOFT Soft. Eng. Notes 30(4), 1-8 (2005)

14. Kraus, S., Wilkenfeld, J., Zlotkin, G.: Multiagent negotiation under time constraints. Artificial Intelligence 75(2), 297-345 (1995) 
15. Fatima, S.S., Wooldridge, M., Jennings, N.R.: Multi-issue negotiation under time constraints. In: Proceedings of the First international Joint Conference on Autonomous Agents and Multiagent Systems (AAMAS 2002), pp. 143-150. ACM, New York (2002)

16. Bouroche, M., Hughes, B., Cahill, V.: Real-time coordination of autonomous vehicles. In: Proceedings of IEEE Conference on Intelligent Transportation Systems (ITSC 2006), pp. 1232-1239. IEEE Computer Society, Los Alamitos (2006)

17. Mock, M., Nett, E.: Real-time communication in autonomous robot systems. In: Proceedings of the Fourth International Symposium on Autonomous Decentralized Systems, pp. 34-41. IEEE Computer Society, Washington (1999)

18. Nwana, H.S., Lee, L.C., Jennings, N.R.: Co-ordination in Multi-Agent Systems. In: Nwana, H.S., Azarmi, N. (eds.) Software Agents and Soft Computing: Towards Enhancing Machine intelligence, Concepts and Applications. LNCS, vol. 1198, pp. 42-58. Springer, Heidelberg (1997)

19. Allen-Williams, M.: Coordination in multi-agent systems, Ph.D thesis, University of Southampton (2005)

20. Ciancarini, P., Omicini, A., Zambonelli, F.: Multiagent System Engineering: The Coordination Viewpoint. In: Jennings, N.R., Lespérance, Y. (eds.) ATAL 1999. LNCS, vol. 1757, pp. 250-259. Springer, Heidelberg (2000)

21. Gelernter, D., Carriero, N.: Coordination languages and their significance. Commun. 35(2), 97-107 (1992)

22. Stankovic, J.A., Abdelzaher, T.F., Lu, C., Sha, L., Hou, J.C.: Real-time communication and coordination in embedded sensor networks. Proceedings of the IEEE 91(7), 1002-1022 (2003) 